\title{
On Transformation Involving Basic Analogue of Multivariable $H$-Function
}

\author{
Dinesh Kumar $\mathbb{D}^{1},{ }^{1}$ Frédéric Ayant, ${ }^{2,3}$ and Jessada Tariboon $\mathbb{D}^{4}$ \\ ${ }^{1}$ Department of Applied Sciences, College of Agriculture, Sumerpur-Pali, Agriculture University of Jodhpur, Jodhpur 342007, \\ Raj, India \\ ${ }^{2}$ College Jean L'herminier, Allée des Nymph eas, 83500 La Seyne-sur-Mer, France \\ ${ }^{3} 411$ Avenue Joseph Raynaud Le parc Fleuri, Bat. B, Six-Fours-les-Plages, 83140 Var, France \\ ${ }^{4}$ Intelligent and Nonlinear Dynamic Innovations Research Center, Department of Mathematics, Faculty of Applied Science, \\ King Mongkut's University of Technology North Bangkok, Bangkok 10800, Thailand
}

Correspondence should be addressed to Jessada Tariboon; jessada.t@sci.kmutnb.ac.th

Received 22 March 2020; Accepted 27 April 2020; Published 28 May 2020

Academic Editor: Serkan Araci

Copyright (C) 2020 Dinesh Kumar et al. This is an open access article distributed under the Creative Commons Attribution License, which permits unrestricted use, distribution, and reproduction in any medium, provided the original work is properly cited.

In this article, fractional order $q$-integrals and $q$-derivatives involving a basic analogue of multivariable $H$-function have been obtained. We give an application concerning the basic analogue of multivariable $H$-function and $q$-extension of the Leibniz rule for the fractional $q$-derivative for a product of two basic functions. We also give the corollary concerning basic analogue of multivariable Meijer's $G$-function as a particular case of the main result.

\section{Introduction and Preliminaries}

The $q$-calculus is not of recent appearance, it was introduced in the twenties of last century. In 1910, Jackson [1] introduced and developed $q$-calculus systematically. The fractional $q$-calculus is the expansion of ordinary fractional calculus in the $q$-theory. Recently, there was a significant work done by many authors in the area of $q$-calculus due to lots of applications in mathematics, statistics, and physics.

Since special functions play significant roles in mathematical physics, it is persuaded to think that some deformation of the ordinary special functions based on the $q$-calculus can also play comparable roles in this area of research. Further, many authors have derived images of various $q$-special functions under fractional $q$-calculus operators; see, for example, [2-7], and may more. The $q$-fractional integrals and derivatives was firstly studied by Al-Salam [8] (see also, [9]). Many researchers have used these operators to evaluate fractional $q$-calculus formulas for various special function, general class of $q$-polynomials, basic analogue of Fox's $H$-function, fractional $q$-calculus formulas for various special function, and etc. One may refer to the recent work $[2-7,10-14]$ on fractional $q$-calculus. Throughout this article, let $\mathbb{Z}, \mathbb{C}, \mathbb{R}, \mathbb{R}_{+}$, and $N$ be the sets of integers, complex numbers, real numbers, positive real numbers, and positive integers, respectively, and let. $\mathbb{N}_{0}:=\mathbb{N} \cup\{0\}$.

The objective of this article is to establish fractional $q$-integral and $q$-derivative of Riemann-Liouville type involving a basic analogue of multivariable $H$-function. We also give an application of $q$-Leibniz formula.

In the $q$-calculus theory, for a real parameter $q \in \mathbb{R}^{+} \backslash\{1\}$, we have a $q$-real number $[a] q$

and $q$-shifted factorial ( $q$-analogue of the Pochhammer symbol) as given by

$$
[a]_{q} \frac{1-q^{a}}{1-q},(a ; q)_{n}=\prod_{i=1}^{n-1}\left(1-a q^{i}\right)(a \in \mathbb{R}, n \in \mathbb{N} \cup\{\infty\})
$$


The $q$-Factorial function is defined by

$$
(a ; q)_{n}=\left\{\begin{array}{c}
1, \text { if } n=0 \\
\prod_{k=0}^{n=1}\left(1-a q^{k}\right), \text { if } n \in \mathbb{N} .
\end{array}\right.
$$

Its extension is

$$
(a ; q)_{n}=\frac{(a ; q) \infty}{\left(a q^{n} ; q\right) \infty}(n \in \mathbb{Z})
$$

which can be elaborated to $n=\alpha \in \mathbb{C}$, given by

$$
(a ; q)_{\alpha}=\frac{(a ; q) \infty}{\left(a q^{n} ; q\right) \infty}(\alpha \in \mathbb{C} ;|q|<1)
$$

where the principal value of $q^{\alpha}$ is taken.

In terms of the $q$-gamma function, (2) can be written as

$$
(a ; q)_{n}=\frac{\Gamma_{q}(a+n)(1-q)^{n}}{\Gamma_{q}(a)}(a \in \mathbb{R} \backslash\{0,-1,-2,\}),
$$

where the q-gamma function [15] is given by

$\Gamma_{q}(a)=\frac{(q ; q)_{\infty}(1-q)^{1-a}}{\left(q^{a} ; q\right)_{\infty}}=\frac{(q ; q)_{a-1}}{(1-q)^{a-1}}(|q|<1 ; a \in \mathbb{R} \backslash\{0,-1,-2\})$,

obviously,

$$
\Gamma_{q}(a+1)=[a]_{q} \Gamma_{q}(a) q !(|q|<1) .
$$

The $q$-analogue of the familiar Riemann-Liouville fractional integral operator of a function $f(x)$ is defined by (see Al-Salam [8])

$$
I_{q}^{\mu}\{f(x)\}=\frac{1}{\Gamma_{q}(\mu)} \int_{0}^{x}(x-t q)_{\mu-1} f(t) d_{q} t(\Re(\mu)>0,|q|<1),
$$

also $q$-analogue of the power function is defined as

$$
(x-y)_{v}=x^{v}\left(\frac{y}{x} ; q\right)_{v}=x^{v} \prod_{n=0}^{\infty}\left[\frac{1-(y / x) q^{n}}{1-(y / x) q^{n+v}}\right], x \neq 0 .
$$
[15])

The basic integral is given by (see Gasper and Rahman

$$
\int_{0}^{x} f(t) d_{q} t=x(1-q) \sum_{k=0}^{\infty} q^{k} f\left(x q^{k}\right)
$$

The equation (8) in conjunction with (10) yield the following series representation of the Riemann-Liouville fractional integral operator

$$
I_{q}^{\mu} f(x)=\frac{x^{\mu}(1-q)}{\Gamma_{q}(\mu)} \sum_{k=0}^{\infty} q^{k}\left[1-q^{k+1}\right]_{\mu-1} f\left(x q^{k}\right) .
$$

In particular, for $f(x)=x^{\lambda-1}$, we have [4]

$$
I_{q}^{\mu}\left(x^{\lambda-1}\right)=\frac{\Gamma_{q}(\lambda)}{\Gamma_{q}(\lambda+\mu)} x \lambda+\mu-1(\mathfrak{R}(\lambda+\mu)>0) .
$$

\section{Basic Analogue of Multivariable $H$-Function}

In this section, we introduce the basic analogue of multivariable $H$-function $[16,17]$, given by the following manner:

$$
\begin{aligned}
& H\left(z_{1}, z_{r} ; q\right)=H_{p, q_{1}^{\prime}: p_{1} q_{1} ; \cdots ; p_{r}, q_{r}}^{0, n: m_{r}, n_{r} ; m_{r} n_{r}}\left(\begin{array}{c|l|l}
z_{1} \\
\cdot & \left(a_{j} ; \alpha_{j}^{(r)} \cdots, \alpha_{j}^{(r)}\right)_{1, p}:\left(c_{j}^{\prime}, \gamma^{\prime}\right)_{1, p_{1}}, \cdots,\left(c_{j}^{(r)}, \gamma_{j}^{(r)}\right)_{1, p_{r}} \\
\cdot & \left(b_{j} ; \beta_{j}^{\prime}, \cdots, \beta_{j}^{(r)}\right)_{1, q^{\prime}}:\left(d_{j}^{\prime}, \delta^{\prime}\right)_{1, q_{1}}, \cdots,\left(d_{j}^{(r)}, \delta_{j}^{(r)}\right)_{1, q_{1}} \\
z_{r}
\end{array}\right) \\
& =\frac{1}{(2 \pi \omega)^{r}} \int_{L 1} \cdots \int_{L_{r}} \pi^{r} \phi\left(s_{1}, \cdots, s_{r} ; q\right) \prod_{i=1}^{r} \theta_{i}\left(s_{i} ; q\right) x_{1}^{s_{1}} \cdots x_{r}^{s_{r}} d_{q} s_{1} \cdots d_{q} s_{r},
\end{aligned}
$$


where $\omega=\sqrt{-1}$, and

$$
\begin{aligned}
\phi\left(s_{1}, \cdots, s_{r} ; q\right) & =\frac{\prod_{j=1}^{n} G\left(q^{1-\alpha_{j}+\sum_{i=1}^{r} \alpha_{j}^{(i)} s_{i}}\right)}{\prod_{j=n+1}^{p} G\left(q^{a_{j}-\sum_{i}^{r} \alpha_{j}^{(i)} s_{i}}\right) \prod_{j=1}^{q^{\prime}} G\left(q^{1-b_{j}+\sum_{i=1}^{r} \beta_{j}^{(i)} s_{i}}\right)}, \\
\theta_{i}\left(s_{1} ; q\right) & =\frac{\prod_{j=1}^{m_{i}} G\left(q^{d_{j}^{(i)}-\delta_{j}^{(i)} s_{i}}\right) \prod_{j=1}^{n_{i}} G\left(q^{1-c_{j}^{(i)}+\gamma_{j}^{(i)} s_{i}}\right)}{\prod_{j=m_{i}+1}^{q_{i}} G\left(q^{1-d_{j}^{(i)}+\delta_{j}^{(i)} s_{i}}\right) \prod_{j=n_{i}+1}^{p_{i}} G\left(q^{c_{j}^{(i)}+\gamma_{j}^{(i)} s_{i}}\right) G\left(q^{1-s_{i}}\right) \sin \pi s_{i}},
\end{aligned}
$$

here, $i=1, \cdots, r$ and

$$
G\left(q^{a}\right)=\left[\prod_{n=0}^{\infty}\left(1-q^{a+n}\right)\right]^{-1}=\frac{1}{\left(q^{a} ; q\right)_{\infty}} .
$$

The integers $n, p, q, m_{i}, n_{i}, p_{i}, q_{i}$ are constrained by the inequalities $0 \leq n \leq p, 0 \leq q^{\prime}, 1 \leq m_{i} \leq q_{i}$ and $0 \leq n_{i} \leq p_{i}, i=1$, $\cdots, r$. The poles of integrand are assumed to be simple. The quantities $a_{j}(j=1, \cdots, p) ; c_{j}^{(i)}\left(j=1, \cdots, p_{i}\right) ; b_{j}\left(j=1, \cdots, q^{\prime}\right) ; d_{j}^{(i)}$ $\left(j=1, \cdots, q_{i}, i=1, \cdots, r\right)$ are complex numbers and the following quantities $\alpha_{j}^{(i)}(j=1, \cdots, p) ; \gamma_{j}^{(i)}\left(j=1, \cdots, p_{i}\right) ; \beta_{j}^{(i)}\left(j=1, \cdots, q^{\prime}\right)$; $\delta_{j}^{(i)}\left(j=1, \cdots, q_{i}, i=1, \cdots, r\right)$ are positive real numbers.
The contour $L_{i}$ in the complex $s_{i}$-plane is of the MellinBarnes type which runs from $-\omega \infty$ to $\omega \infty$ with indentations, if necessary to make certain that all the poles of $G\left(q^{d_{j}^{(i)}+\delta_{j}^{(i)} s_{i}}\right)$ $\left(j=1, \cdots, m_{i}\right)$ are separated from those of, $G\left(q^{1-c_{j}^{(i)}+\gamma_{j}^{(i)} s_{i}}\right)$ $\left(i=1, \cdots, n_{i}\right) G\left(q^{1-a_{j}+\sum_{i=1}^{r} \alpha_{j}^{(i)} s_{i}}\right)(j=1, \cdots, n)$. For large values of $\left|s_{i}\right|$, the integrals converge if $\mathfrak{R}\left(s \log \left(z_{i}\right)-\log \sin \pi s_{i}\right)<0$ $(i=1, \cdots, r)$.

If the quantities $\alpha_{j}^{(i)}(j=1, \cdots, p) ; \gamma_{j}^{(i)}\left(j=1, \cdots, p_{i}\right) ; \beta_{j}^{(i)}$ $\left(j=1, \cdots, q^{\prime}\right) ; \delta_{j}^{(i)}\left(j=1, \cdots, q_{i}\right)=1$ for $i=1, \cdots, r$, then the basic analogue of multivariable $H$-function reduces in basic analogue of multivariable Meijer's $G$-function defined by Khadia and Goyal [18], we obtain

$$
\begin{aligned}
G\left(z_{1}, \cdots, z_{r} ; q\right) & =G_{p, q^{\prime}: p_{1} q_{1} ; \cdots ; p_{r}, q_{r}}^{0, n: n_{r}, \cdots ; m_{r}}\left(\begin{array}{c|c}
z_{1} \\
\cdot & \left(a_{j}\right)_{p}:\left(c_{j}^{\prime}\right)_{p_{1}}, \cdots,\left(c_{j}^{(r)}\right)_{p_{r}} \\
\cdot & \left(b_{j}\right)_{q^{\prime}}:\left(d_{j}^{\prime}\right)_{q_{1}}, \cdots,\left(d_{j}^{(r)}\right)_{q_{1}} \\
z_{r}
\end{array}\right. \\
& =\frac{1}{(2 \pi \omega)^{r}} \int_{L 1} \cdots \int_{L_{r}} \pi^{r} v\left(s_{1}, \cdots, s_{r} ; q\right) \prod_{i=1}^{r} v_{i}\left(s_{i} ; q\right) z_{1}^{s_{1}} \cdots z_{r}^{s_{r}} d_{q_{1}} s_{1} \cdots d_{q_{r}},
\end{aligned}
$$

where

$$
\begin{aligned}
v\left(s_{1}, \cdots, s_{r} ; q\right) & =\frac{\prod_{j=1}^{n} G\left(q^{1-\alpha_{j}+\sum_{i=1}^{r} s_{i}}\right)}{\prod_{j=n+1}^{p} G\left(q^{a_{j}-\sum_{i=1}^{r} s_{i}}\right) \prod_{j=1}^{q^{\prime}} G\left(q^{1-b_{j}+\sum_{i=1}^{r} s_{i}}\right)}, \\
v\left(s_{1} ; q\right) & =\frac{\prod_{j=1}^{m_{i}} G\left(q^{d_{j}^{(i)}-s_{i}}\right) \prod_{j=1}^{n_{i}} G\left(q^{1-c_{j}^{(i)}+s_{i}}\right)}{\prod_{j=m_{i}+1}^{q_{i}} G\left(q^{1-d_{j}^{(i)}+s_{i}}\right) \prod_{j=n_{i}+1}^{p_{i}} G\left(q^{1-c_{j}^{(i)}-s_{i}}\right) G\left(q^{1-s_{i}}\right) \sin \pi s_{i}},
\end{aligned}
$$

where $i=1, \cdots, r$; the integers $n, p, q, m_{i}, n_{i}, p_{i}, q_{i}$ are constrained by the inequalities $0 \leq n \leq p, 0 \leq q^{\prime}, 1 \leq m_{i} \leq q_{i}$ and $0 \leq n_{i} \leq p_{i}, i=1, \cdots, r$. The poles of integrand are assumed to be simple. The quantities $\alpha_{j}^{(i)}(j=1, \cdots, p) ; c_{j}^{(i)}\left(j=1, \cdots, p_{i}\right)$; $b_{j}\left(j=1, \cdots, q^{\prime}\right) ; d_{j}^{(i)}\left(j=1, \cdots, q_{i} ; i=1, \cdots, r\right) \quad$ are complex numbers.

The contour $L_{i}$ in the complex $s_{i}$-plane is of the Mellin-Barnes type which runs from $-\omega \infty$ to $\omega \infty$ with indentations, if necessary to ensure that all the poles of $G\left(q^{d_{j}^{(i)}+\delta_{j}^{(i)} s_{i}}\right)\left(j=1, \cdots, m_{i}\right)$ are separated from 
those of $G\left(q^{1-c_{j}^{(i)}+s_{i}}\right)\left(i=1, \cdots, n_{i}\right) G\left(q^{1-a_{j}+\sum_{i=1}^{r} s_{i}}\right)(j=1, \cdots, n)$. For large values of $\left|s_{i}\right|$, the integrals converge if $\mathfrak{R}\left(s \log \left(z_{i}\right)-\right.$ $\left.\log \sin \pi s_{i}\right)<0(i=1, \cdots, r)$.

\section{Main Results}

In this section, we establish two fractional $q$-integral formulas about the basic analogue of multivariable $H$-function.

Let

$$
\begin{aligned}
& U=m_{1}, n_{1} ; \cdots ; m_{r}, n_{r} ; V=p_{1}, q_{1} ; \cdots ; p_{r}, q_{r} \\
& A=\left(a_{j} ; \alpha_{j}^{\prime}, \cdots, \alpha_{j}^{(r)}\right)_{1, p} ; B=\left(c_{j}^{\prime}, \gamma^{\prime}\right)_{1, p_{1}}, \cdots,\left(c_{j}^{(r)}, \gamma_{j}^{(r)}\right)_{1, p_{r}} \\
& C=\left(b_{j} ; \beta_{j}^{\prime}, \cdots, \beta_{j}^{(r)}\right)_{1, q^{\prime}} ; D=\left(d_{j}^{\prime}, \delta^{\prime}\right)_{1, q_{1}}, \cdots,\left(d_{j}^{(r)}, \delta_{j}^{(r)}\right)_{1, q_{r}} .
\end{aligned}
$$

Theorem 1. Let $\mathfrak{R}(\mu)>0,|q|<1$, the Riemann-Liouville fractional q-integral of a product of two basic functions exists, and we have

$$
\begin{aligned}
& I_{q}^{\mu}\left\{x^{\lambda-1} H_{p, q: V}^{0, n: U}\left(\begin{array}{cc|c}
z_{1} x^{P 1} & \\
\cdot & & \\
\cdot & & A: B \\
& & \\
z_{1} x^{\mathrm{Pr}} &
\end{array}\right)\right\} \\
& =(1-q)^{\mu} x^{\lambda+\mu-1} H_{p+1, q^{\prime}+1: V}^{0, n+U}\left(\begin{array}{c|c|l}
z_{1} x^{P 1} & \\
\cdot & ; q \mid \begin{array}{l}
\left(1-\lambda ; p_{1}, \cdots, p_{r}\right) A: B \\
C\left(1-\lambda-\mu ; p_{1}, \cdots, p_{r}\right): D \\
\cdot
\end{array} \\
z_{1} x^{\mathrm{Pr}} &
\end{array}\right) \text {, }
\end{aligned}
$$

where $p_{i} \in \mathbb{N}, \mathfrak{R}\left(s \log \left(z_{i}\right)-\log \sin \pi s_{i}\right)<0$ for $i=1, \cdots, r$.

Proof. To prove the result (19), we consider the left hand side of equation (19) (say I) and take the definitions (8) and (13) into account, we have

$$
\begin{aligned}
\mathcal{I}= & \frac{1}{\Gamma_{q}(\alpha)} \int_{0}^{x}(x-y q)_{\alpha-1} \frac{y^{\lambda-1}}{(2 \pi w)^{r}} \int_{L_{1}} \cdots \int_{L_{r}} \pi^{r} \phi\left(s_{1}, \cdots, s_{r} ; q\right) \\
& \cdot \prod_{i=1}^{r} \theta_{i}\left(s_{i} ; q\right) \times z_{1}^{s_{r}} \cdots z_{r}^{s_{r}} y^{\rho_{1} s_{1}+\cdots \rho_{r} s_{r}} d_{q} s_{1} \cdots d_{q} s_{r} d_{q} y .
\end{aligned}
$$

Interchanging the order of integrations which is permissible under the given conditions, we obtain

$$
\begin{aligned}
\mathcal{I}= & \frac{1}{\Gamma_{q}(\alpha)(2 \pi w)^{r}} \int_{L_{1}} \cdots \int_{L_{r}} \pi^{r} \phi\left(s_{1}, \cdots, s_{r} ; q\right) \prod_{i=1}^{r} \theta_{i}\left(s_{i} ; q\right) z_{1}^{s_{r}} \cdots z_{r}^{s_{r}} \\
& \times \int_{0}^{x}(x-y q)_{\alpha-1}\left\{y^{\rho_{1} s_{1}+\cdots \rho_{r} s_{r}+\lambda-1}\right\} \mathrm{d}_{q} y \mathrm{~d}_{q} s_{1} \cdots \mathrm{d}_{q} s_{r} .
\end{aligned}
$$

The above equation writes

$$
\begin{aligned}
\mathcal{I}= & \frac{1}{(2 \pi w)^{r}} \int_{L_{1}} \cdots \int_{L_{r}} \pi^{r} \phi(s, t ; q) \theta_{1}(s ; q) \cdots \theta_{r}(s ; q) z_{1}^{s_{1}} \cdots z_{r}^{\rho_{r}} \\
& \times I_{q}^{\mu}\left\{x^{\rho_{1} s_{1}+\cdots+\rho_{r} s_{r}+\lambda-1}\right\} \mathrm{d}_{q} s_{1} \cdots \mathrm{d}_{q} s_{r} .
\end{aligned}
$$

Now using the result (12), then the equation (22) reduces as

$$
\begin{aligned}
\mathcal{I}= & \frac{1}{(2 \pi w)^{r}} \int_{L_{1}} \cdots \int_{L_{r}} \pi^{r} \phi(s, t ; q) \theta_{1}(s ; q) \cdots \theta_{r}(s ; q) z_{1}^{s_{1}} \cdots z_{r}^{\rho_{r}} \\
& \times \frac{\Gamma_{q}\left(\rho_{1} s_{1}+\cdots \rho_{r} s_{r}+\lambda\right)}{\Gamma_{q}\left(\rho_{1} s_{1}+\cdots \rho_{r} s_{r}+\lambda+\mu\right)} x^{\rho_{1} s_{1}+\cdots \rho_{r} s_{r}+\lambda+\mu-1} .
\end{aligned}
$$

Next, interpreting the $q$-Mellin-Barnes multiple integrals contour in terms of the basic analogue of multivariable $H$-function, then we get the desired result (19).

If we replace $\mu$ by $-\mu$ in Theorem 1 , and use the fractional $q$ - derivative operator defined as

$I_{q}^{-\mu}\{f(x)\}=D_{x, q}^{\mu} f(x)=\frac{1}{\Gamma_{q}(-\mu)} \int_{0}^{x}(x-t q)_{-\mu-1} f(t) \mathrm{d}_{q} t(\Re(\mu)<0)$,

and power function formula

$$
D_{x, q}^{\mu}\left\{x^{\lambda-1}\right\}=\frac{\Gamma_{q}(\lambda)}{\Gamma_{q}(\lambda-\mu)} x^{\lambda-\mu-1}(\lambda \neq-1,-2, \cdots),
$$

then we have the following result: 
Theorem 2. Let $\mathfrak{R}(\mu)>0,|q|<1$ the Riemann Liouville fractional q-derivative of a product of two basic functions exists, and given by

$$
\begin{aligned}
& D_{x, q}^{\mu}\left\{x ^ { \lambda - 1 } H _ { p , q ^ { \prime } : V } ^ { 0 , n : U } \left(\begin{array}{cc}
z_{1} x^{\rho_{1}} & \\
\cdot & \\
\cdot & ; q \mid \begin{array}{l}
A: B \\
\cdot
\end{array} \\
C: D \\
z_{r} x^{\rho_{r}}
\end{array}\right.\right. \\
& =(1-q)^{-\mu} x^{\lambda-\mu-1} H_{p+1, q^{0}+1: V}^{0,1: U}\left(\begin{array}{c|c|c}
z_{1} x^{\rho_{1}} & \\
\cdot & ; q \mid \begin{array}{l}
\left(1-\lambda ; \rho_{1}, \cdots, \rho_{r}\right) A: B \\
C\left(1-\lambda+\mu ; \rho_{1}, \cdots, \rho_{r}\right): D \\
\cdot
\end{array} \\
z_{r} x^{\rho_{r}} &
\end{array}\right) \text {, }
\end{aligned}
$$

where $\rho_{i} \in \mathbb{N}, \mathfrak{R}\left(s \log \left(z_{i}\right)-\log \sin \pi s_{i}\right)<0$ for $i=1, \cdots, r$.

Proof. The proof of result asserted by Theorem 2 runs parallel to that of Theorem 1.

The details are, therefore, being omitted.

\section{Leibniz's Application}

In this section, we give an application concerning the basic analogue of multivariable $H$-function and $q$-extension of the Leibniz rule for the fractional $q$-derivative for a product of two basic functions.

We have the $q$-extension of the Leibniz rule for the fractional $q$-derivatives for a product of two basic functions in terms of a series involving the fractional $q$-derivatives of the function, in the following manner [9]:

\section{Lemma 3.}

$$
\begin{aligned}
D_{x, q}^{\alpha} & \{W(x) Y(x)\} \\
= & \sum_{n=0}^{\infty} \frac{(-1)^{n} q^{n(n+1) / 2}\left[q^{-\mu} ; q\right]_{n}}{(q ; q)_{n}} D_{x, q}^{\mu-n}\left\{W\left(x q^{n}\right)\right\} D_{x, q}^{n}\{Y(x)\}
\end{aligned}
$$

where $W(x)$ and $Y(x)$ are two regular functions.

Theorem 4. Let $\mathfrak{R}(\mu)<0$, then the Riemann-Liouville fractional q-derivative of a product of two basic function exists and given by

$$
\begin{aligned}
& H_{p+1, q^{\prime}+1: V}^{0, n+1: U}\left(\begin{array}{c|c|c}
z_{1} x^{\rho_{1}} & \\
\cdot & ; q \mid \begin{array}{l}
\left(1-\lambda ; \rho_{1}, \cdots, \rho_{r}\right), A: B \\
C,\left(1-\lambda+\mu ; \rho_{1}, \cdots, \rho_{r}\right): D
\end{array} \\
\cdot & \\
z_{r} x^{\rho_{r}} &
\end{array}\right) \\
& =\sum_{n=0}^{\infty} \frac{(-1)^{n} q^{n \lambda+(n(n-1) / 2)}\left[q^{-\mu} ; q\right]_{n}}{(q ; q)_{n}\left(q^{\lambda} ; q\right)_{n-\mu}} H_{p+1, q^{\prime}+1: V}^{0, n+1: U} \\
& \cdot\left(\begin{array}{cc|c}
z_{1} x^{\rho_{1}} & \\
\cdot & \\
\cdot q \mid \begin{array}{l}
\left(0 ; \rho_{1}, \cdots, \rho_{r}\right) A: B \\
C\left(n ; \rho_{1}, \cdots, \rho_{r}\right): D
\end{array} \\
z_{r} x^{\rho_{r}}
\end{array}\right.
\end{aligned}
$$

where $p_{i} \in \mathbb{N}, \mathfrak{R}\left(s \log \left(z_{i}\right)-\log \sin \pi s_{i}\right)<0$ for $i=1, \cdots, r$.

Proof. For applying $q$-Leibniz rule, we let

$$
W(x)=x^{\lambda-1} \text { and } Y(x) H_{p, q^{\prime}: V}^{0, n: U}\left(\begin{array}{cc|c}
z_{1} x^{\rho_{1}} & & \\
\cdot & & \\
\cdot & ; q \mid \begin{array}{c}
A: B \\
\cdot
\end{array} \\
& & \\
z_{r} x^{\rho_{\mathrm{r}}} &
\end{array}\right) .
$$

By using the lemma 3, we have

$$
\begin{aligned}
& D_{x, q}^{\mu}\left\{x^{\lambda-1} H_{p, q^{\prime}: V}^{0, U}\left(\begin{array}{cc|c}
z_{1} x^{\rho_{1}} & & \\
\cdot & & \\
\cdot & & \\
\cdot & & \\
& & \\
z_{r} x^{\rho_{r}} &
\end{array}\right)\right\} \\
& =\sum_{n=0}^{\infty} \frac{(-1)^{n} q^{n(n-1) / 2}\left[q^{-\mu} ; q\right]_{n}}{(q ; q)_{n}} D_{x, q}^{\mu-n}\left\{\left(x q^{n}\right)^{\lambda-1}\right\} D_{x, q}^{n} \\
& \cdot\left\{H\left(z_{1} x^{\rho_{1}}, \cdots, z_{r} x^{\rho_{r}} ; q\right)\right\} \text {. }
\end{aligned}
$$

Next, by setting $\lambda=1$ and using the Theorem 2, we arrive at 


$$
\begin{aligned}
& D_{x, q}^{n}\left\{H\left(z_{1} x^{\rho_{1}}, \cdots, z_{r} x^{\rho_{r}} ; q\right)\right\} \\
& =(1-q)^{-\mu} x^{-\mu} \times H_{p+1, q^{\prime}+1: V}^{0, n+1:}\left(\begin{array}{cc|c}
z_{1} x^{\rho_{1}} & \\
\cdot & \\
\cdot & ; q \mid \begin{array}{l}
\left(0 ; \rho_{1}, \cdots, \rho_{r}\right) A: B \\
C\left(n ; \rho_{1}, \cdots, \rho_{r}\right): D \\
\cdot \\
z_{r} x^{\rho_{\mathrm{r}}}
\end{array}
\end{array}\right.
\end{aligned}
$$

where $\rho_{i} \in \mathbb{N}, \mathfrak{R}\left(s \log \left(z_{i}\right)-\log \sin \pi s_{i}\right)<0(i=1, \cdots, r)$.

Now, by using (25) and (31) we obtain the desired result (28) after several algebraic manipulations.

\section{Particular Case}

In this section, the basic analogue of multivariable $H$-function reduces in basic analogue of multivariable Meijer's $G$ -function [18].

$$
\begin{aligned}
& \text { Let } \\
& \begin{array}{l}
U=m_{1}, n_{1} ; \cdots ; m_{r}, n_{r} ; V=p_{1}, q_{1} ; \cdots ; p_{r}, q_{r} ; \\
A_{1}=\left(a_{j}\right)_{1, p} ; B_{1}=\left(c_{j}\right)_{1, p_{1}}, \cdots,\left(c_{j}^{(r)}\right)_{1, p_{r}} ; C_{1}\left(b_{j}\right)_{1, q^{\prime}} ; \\
D_{1}=\left(d_{j}\right)_{1 q_{1}}, \cdots,\left(d_{j}^{(r)}\right)_{1, q_{r}} .
\end{array}
\end{aligned}
$$

\section{Corollary 5.}

$$
\begin{aligned}
& D_{x, q}^{\mu}\left\{t^{\lambda-1} G_{p+1, q^{\prime}+1: V}^{0, n+1:}\left(\begin{array}{cc|c}
z_{1} x^{\rho_{1}} & \\
\cdot & & \\
\cdot & ; q \mid \begin{array}{c}
A_{1}: B_{1} \\
C_{1}: D_{1} \\
\cdot \\
z_{r} x^{\rho_{r}}
\end{array} &
\end{array}\right)\right\} \\
& =\sum_{n=0}^{\infty} \frac{(-1)^{n} q^{n \lambda+(n(n-1) / 2)}\left[q^{-\mu} ; q\right]_{n}}{(q ; q)_{n}\left(q^{\lambda} ; q\right)_{n-\mu}} G_{p+1, q^{\prime}+1: V}^{0, n+1: U} \\
& \left(\begin{array}{cc}
z_{1} x^{\rho_{1}} & \\
\cdot & ; q \mid \begin{array}{l}
\left(0 ; \rho_{1}, \cdots, \rho_{r}\right) A_{1}: B_{1} \\
C_{1},\left(n ; \rho_{1}, \cdots, \rho_{r}\right): D_{1} \\
z_{r} x^{\rho_{r}}
\end{array}
\end{array}\right),
\end{aligned}
$$

where $p_{i} \in \mathbb{N}, \mathfrak{R}\left(s \log \left(z_{i}\right)-\log \sin \pi s_{i}\right)<0$ for $i=1, \cdots, r$.

Remark 6. If the basic analogue of multivariable H-function reduces in basic analogue of Srivastava-Daout function [19], then we obtain the results given by Purohit et al. [20].
Remark 7. If the basic analogue of multivariable H-function reduces in basic analogue of $H$-function of two variables defined by Saxena et al. [21], we obtain the result due to Yadav et al. [7]. Further, if the basic analogue of multivariable $H$-function reduces in basic analogue of $H$-function of one variable defined by Saxena et al. [22], then we can easily obtain the similar result.

\section{Conclusion}

In the present article, we have proposed the fractional order $q$ -integrals and $q$-derivatives involving a basic analogue of multivariable $H$-function. The significance of our derived results lies in their diverse generality. By specializing the various parameters as well as variables in the basic analogue of multivariable $H$-function, we can obtain a large number of results involving a remarkably wide range of useful basic functions (or product of such basic functions) of one and several variables. Hence, the derived formulas in this article are most general in character and may reaffirm to be useful in several interesting cases appearing in literature.

\section{Data Availability}

No data were used to support this study.

\section{Conflicts of Interest}

The authors declare that there are no conflicts of interest regarding the publication of this paper.

\section{Acknowledgments}

This research was funded by King Mongkut's University of Technology North Bangkok. Contract no. KMUTNB-61KNOW-021.

\section{References}

[1] F. H. Jackson, “On q-definite integral," Quarterly of Applied Mathematics, vol. 41, pp. 193-203, 1975.

[2] R. K. Saxena, R. K. Yadav, S. L. Kalla, and S. D. Purohit, "Kober fractional $q$-integral operator of the basic analogue of the $H$-function," Revista Técnica de la Facultad de Ingeniería Universidad del Zulia, vol. 28, no. 2, pp. 154-158, 2005.

[3] R. K. Yadav and S. D. Purohit, "On application of Kober fractional $q$-integral operator to certain basic hypergeometric function," Journal of Rajasthan Academy of Physical Sciences, vol. 5, no. 4, pp. 437-448, 2006.

[4] R. K. Yadav and S. D. Purohit, "On applications of Weyl fractional $q$-integral operator to generalized basic hypergeometric functions," Kyungpook National University, vol. 46, pp. 235245, 2006.

[5] R. K. Yadav, S. D. Purohit, and S. L. Kalla, "On generalized Weyl fractional $q$-integral operator involving generalized basic hypergeometric function," Fractional Calculus and Applied Analysis, vol. 11, no. 2, pp. 129-142, 2008.

[6] R. K. Yadav, S. D. Purohit, S. L. Kalla, and V. K. Vyas, "Certain fractional $q$-integral formulae for the generalized basic hypergeometric functions of two variables," Journal of Inequalities and Special Functions, vol. 1, no. 1, pp. 30-38, 2010. 
[7] R. K. Yadav, S. D. Purohit, and V. K. Vyas, "On transformations involving generalized basic hypergeometric function of two variables," Revista Técnica de la Facultad de Ingeniería Universidad del Zulia, vol. 33, no. 2, pp. 176-182, 2010.

[8] W. A. Al-Salam, "Some Fractionalq-Integrals andq-Derivatives," Proceedings of the Edinburgh Mathematical Society, vol. 15, no. 2, pp. 135-140, 1966.

[9] R. P. Agarwal, "Certain fractionalq-integrals andq-derivatives," Mathematical Proceedings of the Cambridge Philosophical Society, vol. 66, no. 2, pp. 365-370, 1969.

[10] L. Galué, "Generalized Weyl fractional $q$-integral operator," Algebras, Groups and Geometries, vol. 26, no. 2, pp. 163-178, 2009.

[11] M. H. Abu-Risha, M. H. Annaby, M. E. H. Ismail, and Z. S. Mansour, "Linear q-difference equations," Zeitschrift für Analysis und ihre Anwendungen, vol. 26, pp. 481-494, 2007.

[12] J. Daiya, J. Ram, and D. Kumar, "The multivariable $H$-function and the general class of Srivastava polynomials involving the generalized Mellin-Barnes contour integrals," Filomat, vol. 30, no. 6, pp. 1457-1464, 2016.

[13] D. Kumar, S. D. Purohit, and J. Choi, "Generalized fractional integrals involving product of multivariable $H$-function and a general class of polynomials," Journal of Nonlinear Sciences and Applications, vol. 9, no. 1, pp. 8-21, 2016.

[14] Z. S. I. Mansour, "Linear sequential $q$-difference equations of fractional order," Fractional Calculus and Applied Analysis, vol. 12, no. 2, pp. 159-178, 2009.

[15] G. Gasper and M. Rahman, Basic Hypergeometric Series, Cambridge Univ Press, Cambridge, 1990.

[16] H. M. Srivastava and R. Panda, "Some expansion theorems and generating relations for the $H$-function of several complex variables," Commentarii Mathematici Universitatis Sancti Pauli, vol. 24, pp. 119-137, 1975.

[17] H. M. Srivastava and R. Panda, "Some expansion theorems and generating relations for the $H$-function of several complex variables II," Commentarii Mathematici Universitatis Sancti Pauli, vol. 25, pp. 167-197, 1976.

[18] S. S. Khadia and A. N. Goyal, "On a generalized function of $n$ variables," Vijnana Parishad Anusandhan Patrika, vol. 13, pp. 191-201, 1970.

[19] H. M. Srivastava and P. W. Karlsson, Multiple Gaussian Hypergeometric Series, Halsted Press (Ellis Horwood Limited, Chichester), John Wiley and Sons, New York, Chichester, Brisbane and Toronto, 1985.

[20] S. D. Purohit, R. K. Yadav, G. Kaur, and S. L. Kalla, "Applications of $q$-Leibniz rule to transformations involving generalized basic multiple hypergeometric functions," Algebras, Groups and Geometries, vol. 25, no. 4, pp. 463-482, 2008.

[21] R. K. Saxena, G. C. Modi, and S. L. Kalla, "A basic analogue of $H$-function of two variables," Revista Técnica de la Facultad de Ingeniería Universidad del Zulia, vol. 10, no. 2, pp. 35-39, 1987.

[22] R. K. Saxena, G. C. Modi, and S. L. Kalla, “A basic analogue of Fox's $H$-function," Revista Técnica de la Facultad de Ingeniería Universidad del Zulia, vol. 6, pp. 139-143, 1983. 